\title{
Sunspot variability and an attempt to predict solar cycle 23 by adaptive filtering
}

\author{
G. K. Rangarajan \\ Indian Institute of Geomagnetism, Colaba, Mumbai 400 005, India \\ (Received July 7, 1997; Revised January 5, 1998; Accepted January 5, 1998)
}

\begin{abstract}
The series of annual mean relative sunspot numbers $\left(R_{\mathrm{z}}\right)$ for 1749-1996 is subjected to the recently developed methodology of Singular Spectrum Analysis (SSA). This technique also enables data-adaptive filtering of the individual spectral components. Low order autoregressive modelling of the components are combined to provide a basis for predicting the solar cycle 23 .

The $R_{\mathrm{z}}$ series is largely dominated by a doublet with periods 11.13 and $10.35 \mathrm{yr}$. close to the nominal solar cycle periodicity, a longer period variation $(\sim 110 \mathrm{yr}$.) which is the envelope of the amplitude maxima and two clusters of periodicities centred around $8 \mathrm{yr}$. and $5.5 \mathrm{yr}$. The solar magnetic cycle has no detectable component. The predicted maximum for cycle 23 will have a magnitude of $\sim 130$ and the epoch of maximum is expected between late 2000 A.D. and early 2001 A.D.
\end{abstract}

\section{Introduction}

Zurich relative sunspot number $\left(R_{\mathrm{z}}\right)$ has long been considered a reliable and an useful index of solar activity. The time series, since the year 1610 , is one of the most extensively analysed data bases. Though a quasi-periodic 11-year oscillation is seen as a permanent feature, the solar activity is known to progress in episodes rather than in a smooth fashion (Dodson and Hedeman, 1972). Harwood and Malin (1977) suggest that the series may be regarded as a sequence of independent and partly overlapping events triggered periodically at intervals of 11 years. Understanding the complex variations of the solar activity and prediction of its change on both a short and a long term basis is becoming increasingly relevant because of our improved knowledge of the Sun-Earth relationship and the role of solar activity on geomagnetic variations, global weather/climate, satellite trajectory, communications etc.

In the analysis of the sunspot data, either a quasi-periodic behaviour is presumed and the components extracted through Fourier transform (Cole, 1973; Cohen and Lintz, 1974 and others) or it is treated as an autoregressive process and the spectrum is evaluated through Maximum Entropy Method (Currie, 1973; Kane and Trivedi, 1985 and others). Some authors describe the shape of the solar cycle in terms of smoothly varying functions with suitable parameters to control the shape of ascent to maximum and the descent to minimum (Hathaway et al., 1994 and references therein). In recent years, approaches through non-linear dynamics and theory of chaos have also been attempted (Mundt et al., 1991; Jinno et al., 1995 and references therein). Some confirmation of a generalized periodic model was given by DeMeyer (1981) with 26 sinusoids, though the model suffered badly for forecast purposes.

Copy right (C) The Society of Geomagnetism and Earth, Planetary and Space Sciences (SGEPSS); The Seismological Society of Japan; The Volcanological Society of Japan; The Geodetic Society of Japan; The Japanese Society for Planetary Sciences.
Apart from the analysis of the sunspot series for quasiperiodic oscillations, many researchers have also attempted to forecast the solar activity in the ensuing cycle. The prediction methods rely either on the fact that the statistical behaviour of the available data base will be preserved in the future also or on the fact that the evolution of the solar cycle, particularly its maximum amplitude, is determined in the declining phase before the beginning of the new cycle. Precursors such as geomagnetic activity in the declining phase have proved fairly successful in predicting the maximum $R_{\mathrm{z}}$ in the following solar cycle. Brown (1992), in an exhaustive analysis of all predictions made for the solar cycle 22 (1986-1996), found that only 10 out of 45 were correct to within $\pm 10 \%$ of the observed peak value and 7 of these were "precursor" type, indicative of the inadequacy of the forecast.

Some features which make prediction of solar activity difficult are (i) the largest three cycles $(21,19$ and 18 in that order) have occurred only recently and the distribution of maximum $R_{\mathrm{z}}$ in a solar cycle has varied randomly (Wilson, 1990a); (ii) there have been changes in the harmonic structure of the record, such as the Maunder minimum (Brown, 1992); (iii) individual cycles, such as cycle 22, exhibit anomalous features not predictable by precursory or statistical methods (Wilson, 1990a).

It is noticed that most of the publications on the prediction of solar activity have emphasized only on the amplitude of the solar cycle and rarely on the forecast of the nature of the complete solar cycle depicting the ascent to maximum and the declining phase leading to the minimum. Our attention was drawn to a very interesting paper by Keppenne and Ghil (1992), who demonstrated the intrinsic ability of the method of adaptive filtering through Singular Spectrum Analysis (SSA) and subsequent prediction through a low-order Autoregressive (AR) process to forecast the southern oscillation index, 3 years ahead. In this paper, we follow the methodology suggested by Keppenne and Ghil (1992) to identify significant spectral components of the sunspot 
series and attempt a prediction of the next solar cycle (No. 23) beginning from its minimum in 1996.

\section{Data and Method of Analysis}

The input consists of the annual mean values of Zurich relative sunspot number $\left(R_{\mathrm{z}}\right)$ since 1749 , as monthly values are available from that time. Prior to 1749 , only annual mean values were estimated but from 1818 daily values of sunspot numbers were beginning to be recorded. Based on the quality of data, the series is classified as, "poor" between 1700 and 1748, "questionable" between 1749 and 1817, "good" from 1818 to 1847 and "reliable" from 1848 onwards (McKinnon, 1987). Wilson (1990a) considers the data for the modern era beginning with solar cycle 10 (1856) "most reliable". Sonett (1982), points out a discontinuity between 1780 and 1800 which can cause discrepancies in the consistency of characteristic oscillations of the sunspot series. In addition to the dominant $11 \mathrm{yr}$. cycle, a $22 \mathrm{yr}$. solar magnetic cycle (SMC) (Hale cycle) which is connected to the polarity inversion of the solar general magnetic field is also associated with solar activity (Dicke, 1988; Attolini et al., 1990). In view of these facts, the sunspot time series is analysed in four distinct groups (i) 1749-1996 (all data), (ii) 1818-1996 (good and reliable data), (iii) 1848-1996 (most reliable data) and (iv) 1749-1996 after reversing the sign of alternate cycles (to incorporate SMC).

We use the recently developed data-adaptive technique, Singular Spectrum Analysis (SSA), which has been described in great detail by Vautard et al. (1992) and a software package developed by their team is now available (Dettinger et al., 1995). They have demonstrated how this method, which is a form of Principal Component Analysis, applied to the lag-correlation structure of the time series, succeeds in isolating trends, quasi-periodic fluctuations etc. through the generation of suitable data-adaptive filters, in suppressing the noise contribution and how the original time series could be closely recreated by the addition of fewer number of principal components.

If $\rho_{0}, \rho_{1}, \rho_{2}, \ldots, \rho_{M-1}$ are the first $M$ auto-correlations of the time series, then the lag-correlation matrix with the Toeplitz structure is generated as

$$
\frac{1}{M}\left[\begin{array}{ccccc}
\rho_{0} & \rho_{1} & \rho_{2} & \cdots & \rho_{M-1} \\
\rho_{1} & \rho_{0} & \rho_{1} & \cdots & \rho_{M-2} \\
\cdots & \cdots & \cdots & \cdots & \cdots \\
\cdots & \cdots & \cdots & \cdots & \cdots \\
\rho_{M-1} & \rho_{M-2} & \cdots & \cdots & \rho_{0}
\end{array}\right]
$$

which is then subjected to an eigen analysis to get the eigen values in descending order and the corresponding eigen vectors.

The greatest advantage of SSA, in contrast to power spectral analysis, is its ability to generate individual time series of the reconstructed components whose evolution with time can be graphically seen for interpretation of these oscillations, their intermittancy and identification of possible causative mechanisms. In addition, the relative contribution of the individual components to the total variance of the time series is obtained so that we can identify and isolate significant trends and quasi-periodic fluctuations from the noisy part of the data.

Maximum Entropy Method (MEM) has been extensively used as a tool for high resolution spectrum analysis (see Marple, 1987 for details and references). Sunspot series has also been analysed using this approach by Currie (1973), Kane and Trivedi (1985), Marple (1987) and several others. Penland et al. (1991) have shown how a spectrum derived from the cumulative addition of the spectra of individual components of SSA leads to a much better resolution and is substantially noise-free even with a low order autoregressive process in comparison to the spectrum derived directly from the original data. Using the autoregressive coefficients $a_{k}(k$ $=1,2, \ldots, M)$, the time series $X_{t}$ can be extrapolated based on past observations as

$$
X_{t}=a_{1} X_{t-1}+a_{2} X_{t-2}+\cdots+a_{M} X_{t-M}+\varepsilon_{t}
$$

where $\varepsilon_{t}$ is the residual noise.

Keppenne and Ghil (1992) suggested that the individual principal components can be extrapolated beyond the data length by low order autoregressive coefficients and all predictions can then be combined to generate the amplitude and shape of the time series in the region of extrapolation. They could successfully demonstrate the effectiveness of this approach with the prediction, 36 months ahead, of the southern oscillation index, computed from monthly mean sea level pressure at Darwin and Tahiti.

In the use of SSA, the major constraint is that the window size $(M)$, relative to the total number of data points, must be decided before the spectrum is evaluated. If $M$ is to small, closely spaced frequencies are unlikely to be resolved but the stability of the estimates and statistical confidence level will be better. If $M$ is too large, the statistical significance of the estimated periodicities is compromised. In general, for oscillations longer than the window size, the even and odd eigen vectors would represent overlapping mean and trend respectively. If a quasi-periodic signal is embedded, it manifests as a pair of eigen values of nearly equal magnitude and the corresponding eigen vectors would be in phase quadrature (Vautard et al., 1992).

The annual mean sunspot series for 1749-1996 (shown in Fig. 1) and two other subsets 1818-1996 (good and reliable data) and 1848-1996 (modern era data) as also the sunspot series 1749-1996 with alternate cycles with reversed sign to represent SMC, are subjected to SSA and the reconstructed components are analysed by maximum entropy method (MEM).

As we are aware that the most dominant oscillation in the sunspot series varies in periodicity between 10 and 12 years and solar magnetic cycle (SMC) could possibly be a basic feature, we adopt a window size of 36 (much less than onethird of the data length, the upper limit for $M$ recommended by Vautard et al., 1992). We could well have used higher values of $M$ (upto say 72) but the smaller window size ensures greater stability and provides adequate resolution. Penland et al. (1991) point out that the window size $M$ is related to the desired resolution of frequencies $(\delta f)$ by $M \approx$ $2 / \Delta \delta f$ where $\Delta$ is the sampling interval. For $M=36$, this gives 


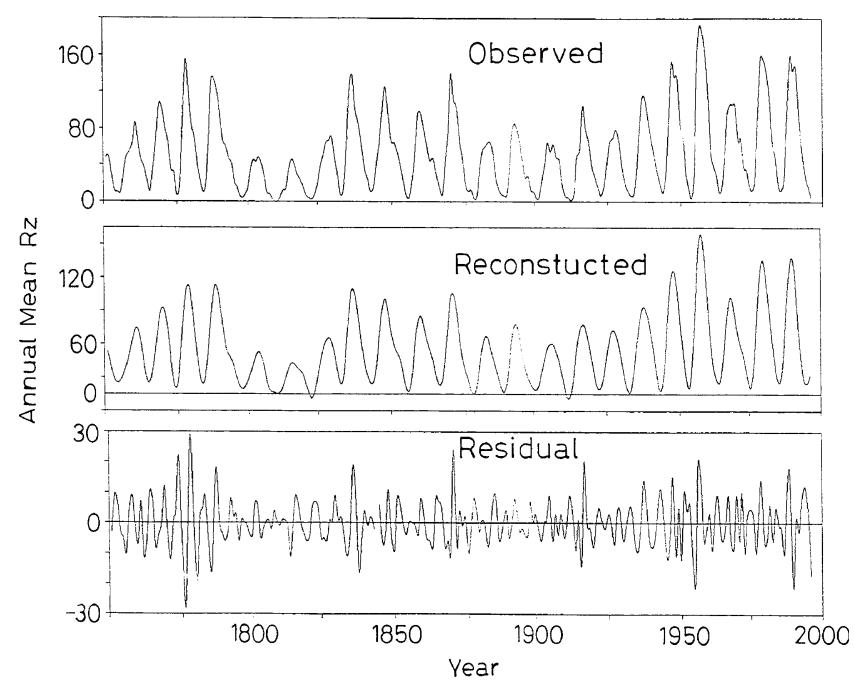

Fig. 1. Annual means of the Zurich relative sunspot number $\left(R_{\mathrm{z}}\right)$ for 1751 to 1996 (top panel) and the corresponding annual values from the cumulative addition of the first twelve reconstructed series (middle panel). The yearly difference between the observed and reconstructed annual mean $R_{\mathrm{z}}$ are also shown (bottom panel).

$\delta f \sim 0.055$. As the spectra reveal later, except for the solar cycle oscillation, other periodicities are well separated showing the adequacy of the choice of $M$ adapted here. The eigen values and the corresponding eigen vectors of the auto-correlation matrix upto a maximum lag of 36 are then computed. The eigen values, in decreasing order, provide an estimate of the significant spectral components in the time series as the largest eigen value corresponds to the maximum spectral density and for finite $M$, all eigen values fall between the maximum and minimum spectral densities (Vautard et al., 1992). The elements of individual eigen vectors constitute the numerical weights of the optimal dataadaptive filter. The principal components, in decreasing order of significance, are generated using these filter weights and end points lost in the computation are reconstructed utilising the formulae given by Vautard et al. (1992).

The reconstructed components (RCs) are spectrally analysed by MEM to (i) derive the dominant spectral line(s) and (ii) estimate the autoregressive coefficients utilising the computer routine outlined in Ulrych and Bishop (1975). Penland et al. (1991) stress that the main point of SSA is that a low order MEM spectrum of the filtered data may be calculated with a resolution comparable to that of a high order MEM spectrum of the original data but without spurious peaks. We find that the reconstructed components are essentially noise-free and, therefore, autoregressive (AR) coefficients with appropriate low order are enough to provide high resolution spectrum and these coefficients can be used to extrapolate the time series using (2). We tried several orders $(5 \%, 7.5 \%, 10 \%$ and $12.5 \%$ of the data length) and found that the change in the AR order does not alter the location of the spectral peaks or the extrapolation significantly. We adopt a fixed choice of 18 for all the analysis even if the length of the time series is different for the three sets.

Each component is extrapolated upto 13 values beyond 1996 covering the years 1997 to 2009 . The reliability of prediction will naturally decrease as one moves farther away

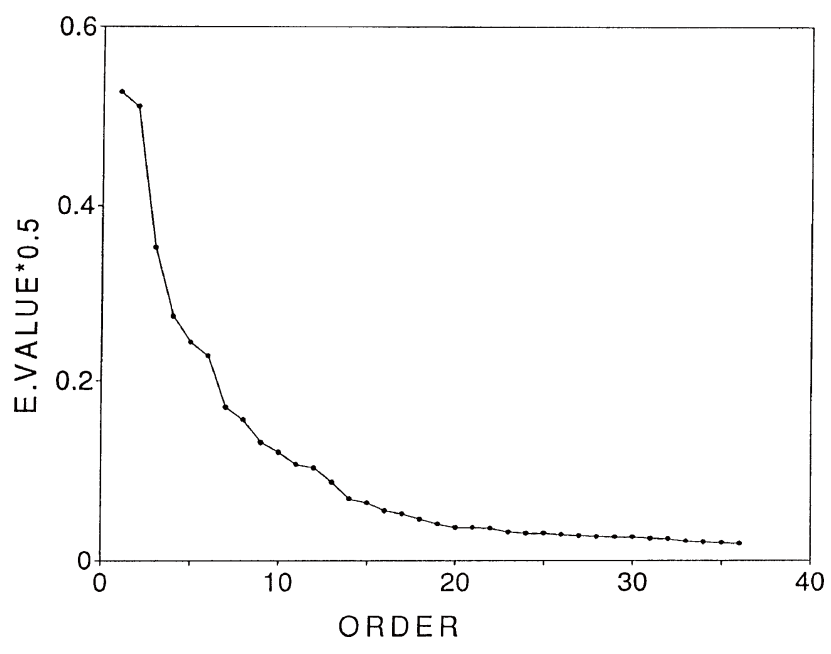

Fig. 2. Singular spectrum (square root of the eigen values in descending order) of $R_{\mathrm{z}}$ for the period 1749-1996.

in time as the predicted value is used for later forecast rather than the actual observations. To minimize the error due to this source, we have modified the approach of Keppenne and Ghil (1992) as follows:

The time series (say) 1749 to 1996 is used to generate anticipated annual sunspot values from 1997 to 2009 . Next, the series 1749-1995, truncated by one end point is used to generate data for 1996 to 2008 and so on till we get predicted values for 1985-1997 from the data for 1749-1984. The 13 individual values in each extrapolation are weighed in decreasing order $(13 / 13,12 / 13, \ldots, 1 / 13)$ so that for the first analysis 1997 will be weighed by unity, for the second run by $12 / 13$ etc. and last run by $1 / 13$. These weighted values for each year are then added, averaged and normalized for unit weight. It is to be noted that the number of values averaged in this process decreases from 13 for the first year (1997) to 1 for the last year 2009. The stability of prediction increases significantly by this approach in comparison with direct prediction using all the data in one step. The window size and the choice of the number of significant RCs play a crucial role in the "direct" prediction whereas their importance, particularly of the window size, is diminished in the "average" prediction approach. We retain for forecast, all the RCs with relative variance in excess of $1 \%$ so that cumulative variance of the reconstructed component is more than $95 \%$.

\section{Results and Discussion}

The presentation of the results and discussions are based on the analysis of the entire series covering the period 1749 to 1996, as the results for this the other subsets and the forecast thereof do not differ significantly. We are also guided by the observation of Mundt et al. (1991) that the "questionable" data (1749-1817) cover less than a quarter of the total and its inclusion does not corrupt or alter the conclusion significantly.

The singular spectrum derived as the square root of the eigen values, in descending order, is shown in Fig. 2. The spectrum defines a clear "noise floor" after a value of 20 . This is gratifying because according to Penland et al. (1991), 

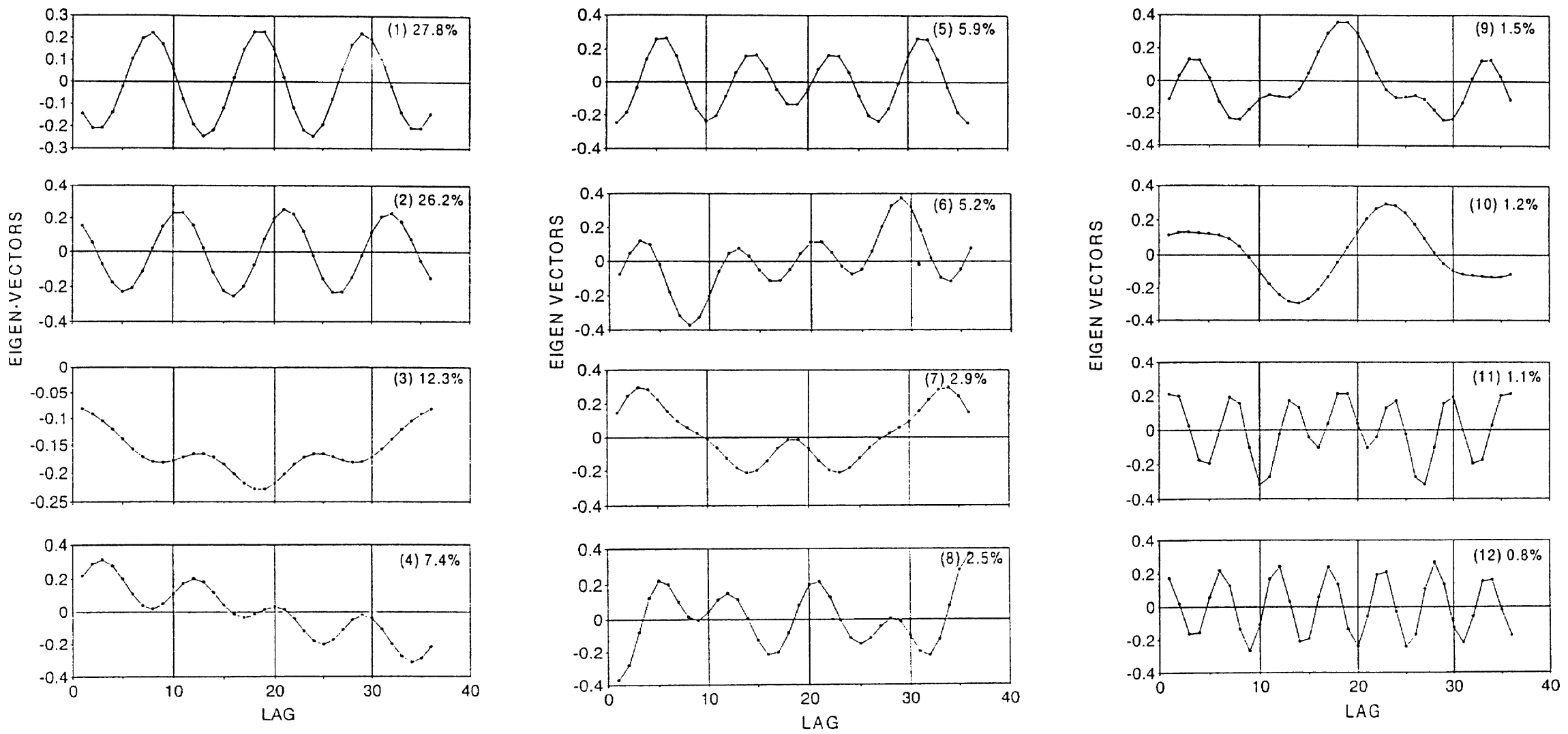

Fig. 3. First 12 eigen vectors of the auto-correlation matrix of the $R_{\mathrm{Z}}$ series with a maximum lag of 36 . Percentage variance accounted for by individual vectors is also shown. 

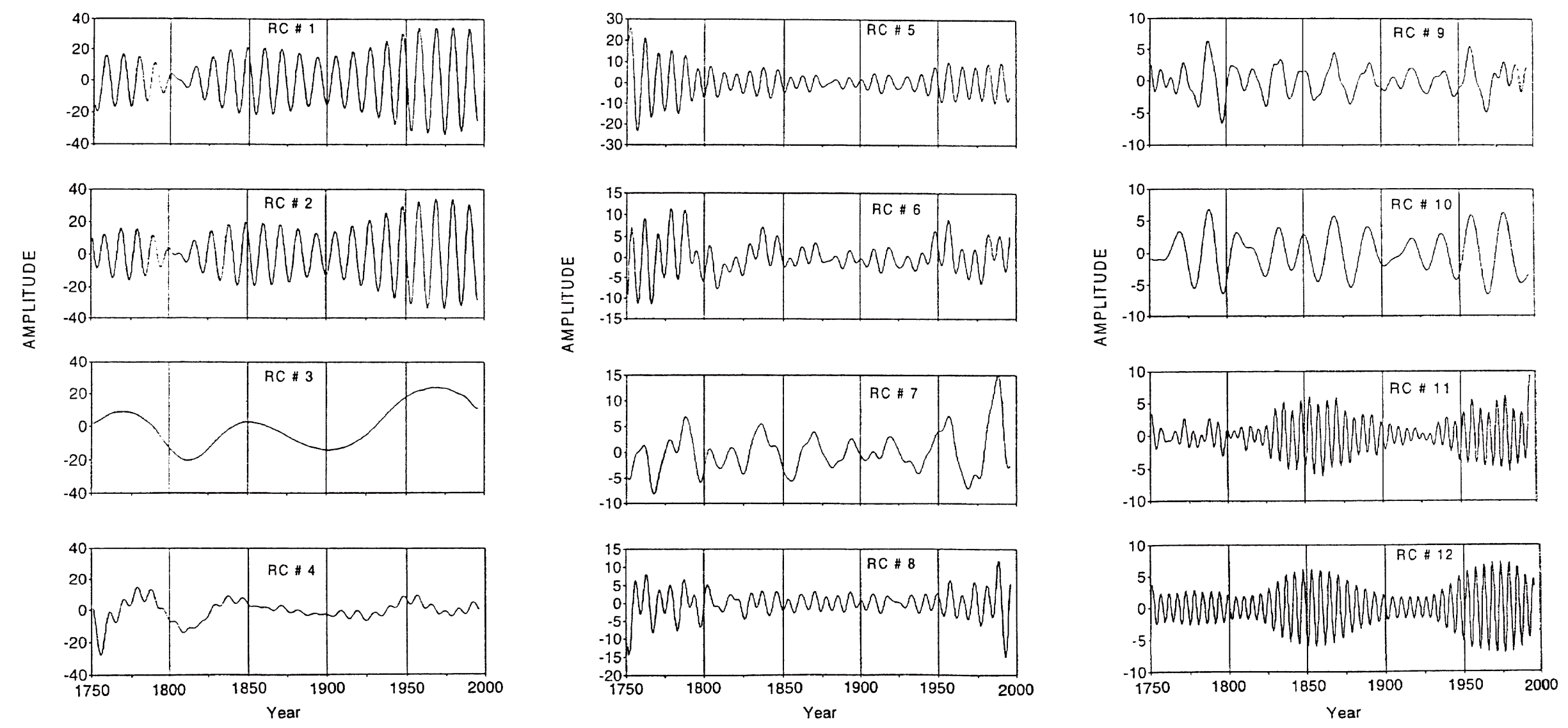

Fig. 4. Reconstructed Components (RCs) of the time series by application of the digital filter with numerical weights provided by the normalized eigen vectors of Fig. 3. 

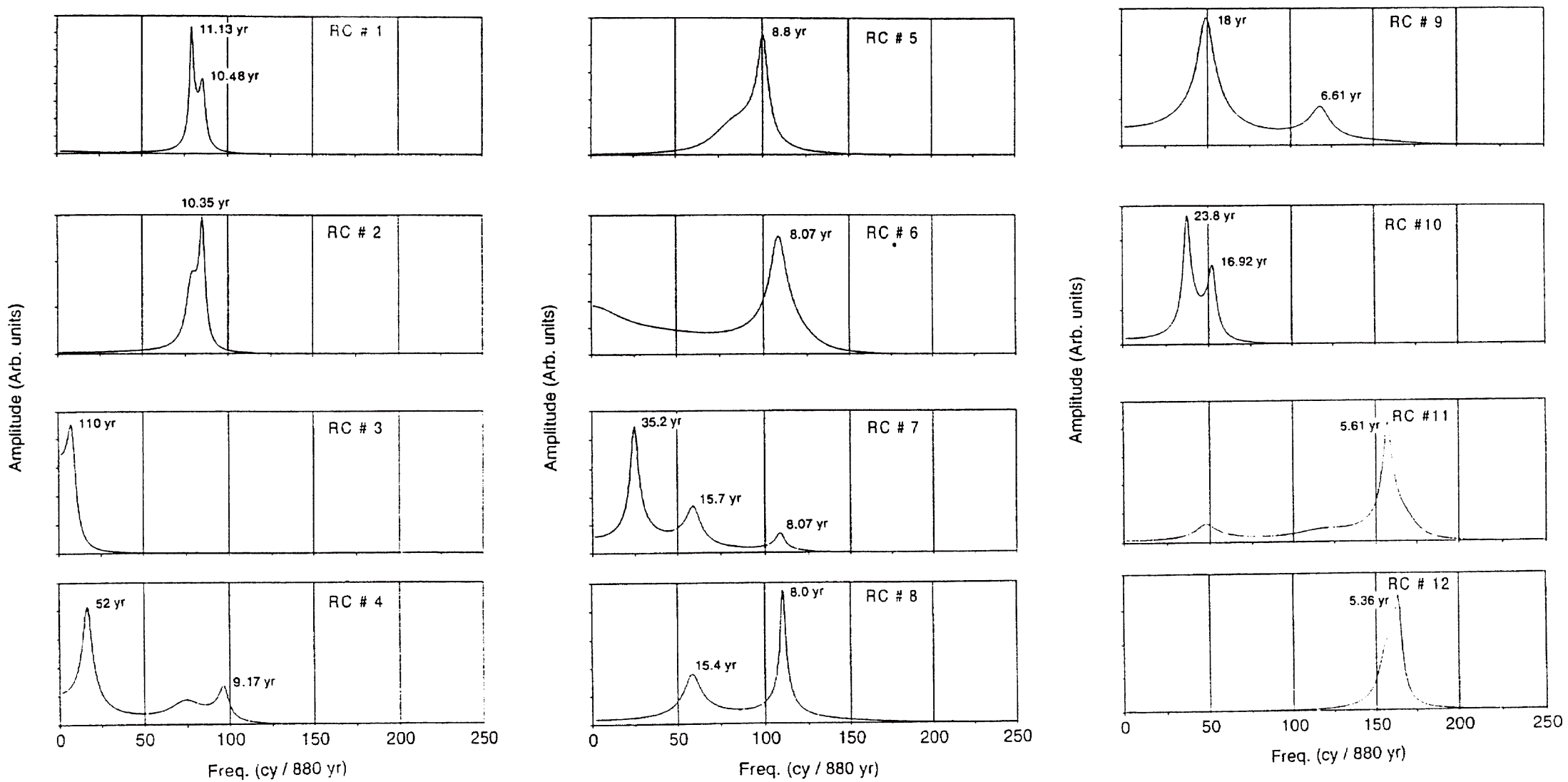

Fig. 5. Maximum Entropy Spectra of the 12 individual RCs with autoregressive coefficients of order 18 
adaptive filtering works best if the graph of SSA eigen value versus index has a flat noise level. One can also notice the absence of eigen-pairs in the spectrum indicating, in turn, the absence of clearly isolated periodic oscillations (Vautard et al., 1992). However, the eigen values corresponding to the pairs of indices 1 and 2, 5 and 6 are fairly close to each other. But, as the corresponding spectra reveal, they do identify separate components. The first 12 eigen values account for $95 \%$ of the total variance.

The first twelve eigen vectors corresponding to the significant eigen values are shown in Fig. 3. The relative contribution of each vector is also shown. The cumulative sum of the first 12 reconstructed components and the mean value is shown in Fig. 1 along with the original series and the difference between the two. The close match between actual observations and the generated series highlights the efficacy of the method of SSA and validity of our choice of truncation. It also highlights the possible margin of error due to the noisy components, not resolved by SSA, present in the data and which cannot be reasonably modelled. The dominant solar cycle oscillation (vectors 1 and 2) accounts for more than $50 \%$. Vectors 3 and 4 (even and odd respectively) describe fluctuations whose periodicity should be outside the range of the viewing window, whereas all others will relate to oscillations with periods less than 36 years. The 36 values of the each eigen vector are next used as numerical weights to generate the filtered and reconstructed time series. The first 12 reconstructed components (RCs) are shown in Fig. 4. The corresponding high resolution MEM spectra using AR order 18 are shown in Fig. 5, to bring out the fact that the noise is largely eliminated and each component has mostly one dominant oscillation.

Both RCs 1 and 2 depict the nominal solar cycle variation (22 cycles in 246 years with an average period of 11.18 years, as seen in Fig. 3). As the first and second eigen values are nearly equal and their corresponding eigen vectors in apparent phase quadrature, it is quite likely that they together represent a quasi-periodicity of $\sim 11 \mathrm{yr}$. Vautard et al. (1992) suggest two natural criteria for verification of this fact: (i) the two components must be spectrally localized around the same frequency $f$ with $2 M \delta f<0.75$ and (ii) at least two thirds of the variance at the peak frequency must be described by the pair. These two criteria appear to be well met suggestive of a single quasi-periodicity of $\sim 11 \mathrm{yr}$. To check whether the phase quadrature is preserved in the reconstructed components 1 and 2, we depict, in Fig. 6, the lagged cross-correlation between the two series. The peak correlation occurs at lag 0 and not between 2 and $3(\sim 11 / 4)$ as should be expected if there were phase quadrature between $\mathrm{RC} 1$ and RC2, the even and odd parts of the same oscillation. In such a case, the magnitude of the correlation should not diminish with increased lag. Contrary to this, we see in Fig. 6 progressively decreasing magnitude of the correlation. The peak value at lag 0 and the diminishing progression of the magnitude of the correlation can, hence, be considered due to the fact that two oscillations, nearly in phase and with slightly different periods are involved. The relevant spectra in Figs. 5(1) and 5(2) clearly isolate the two periods separately as 11.13 and $10.35 \mathrm{yr}$. The inference here is in conformity with the results of MacDonald (1989) who found two strong peaks in the periodogram of sunspot series at frequencies 0.09059 and 0.09394 cpy (11.04 and $10.64 \mathrm{yr}$. periodicities) and the finding of Rabin et al. (1986) that all solar cycles occurred in strings during which the period remained longer or shorter than the sample mean of 132 mon. and a possible gap between 127 and 134 mon.

The longer-period variation in solar activity, brought out by RC3, appears to match the envelope of the amplitude maximum of RC1 and RC2 quite closely. The corresponding spectrum indicates a quasi-periodicity of 110 years (see Fig. 5) but the separation between the first and second peaks in the RC is about $80 \mathrm{yr}$. while that between the second and

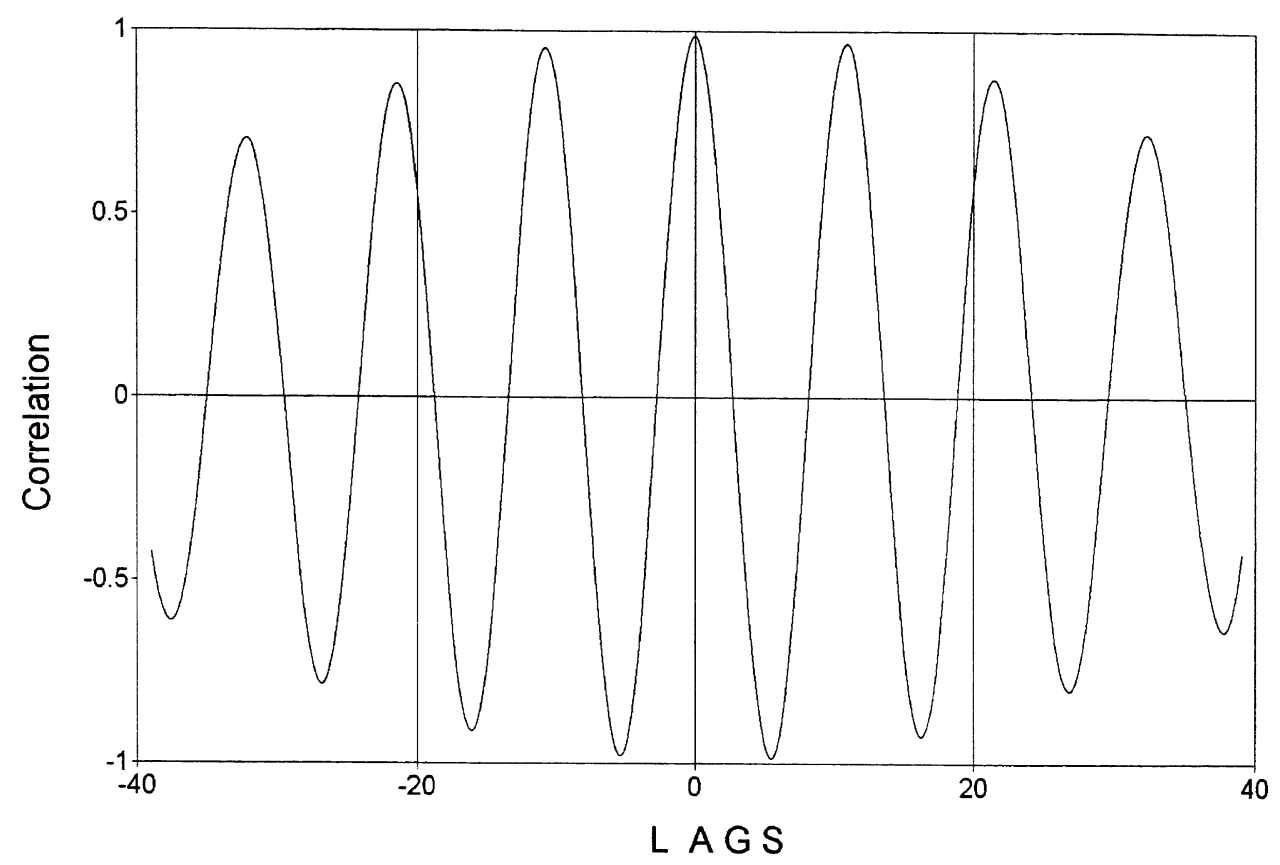

Fig. 6. Cross correlation between RC1 and RC2 at different lags in steps of one year. 
third is about $120 \mathrm{yr}$. This result is consistent with that of Pasricha et al. (1991) who showed that the peak values of the 11-year sunspot cycle constitutes an envelope with a cyclic trend of about 80 years but the envelope does not follow the solar maximum adequately with a period of 80 years.

Though RC1 and RC2 depict the solar cycle variation, they have significantly different associated periodicities of $11.12 \mathrm{yr}$. and $10.35 \mathrm{yr}$. as discussed earlier. MacDonald (1989), identified several frequencies in the spectrum of $R_{\mathrm{z}}$ but suggested that almost all of them can be expressed in terms of low order integer multiples of three fundamental periods of $11.04,10.64$ and 98.33 yr. Our results also clearly demonstrate the fact that the first three significant oscillations, accounting for more than two-thirds of the total variance, have periodicities in the same range. In view of the superiority of this data adaptive methodology and the efficiency of the technique to provide high resolution and noisefree spectrum with low order AR model, we can now assert that at least two of the fundamental periods in $R_{\mathrm{z}}$ are 11.13 and $10.35 \mathrm{yr}$. with the third one near $110 \mathrm{yr}$. Rabin et al. (1986) suggest that the solar dynamo has two modes with different mean periods and also that in the shorter (longer) period mode, the amplitude grows (decays) leading to a long-term trend. Such a relationship between the length of the cycle and the rise and fall of the amplitude envelope was later confirmed by Wilson (1990b). Our results are once again in conformity with these observations.

The cyclic behaviour, with the two periods 11.13 and $10.35 \mathrm{yr}$. is distorted only for a brief interval centred on 1800 A.D. It may be inferred that discontinuity in the sunspot series, pointed out by Sonett (1982) is brought about by only the break-down in its $11 \mathrm{yr}$. cyclicity, as all the other reconstructed components do not exhibit this feature.

Other quasi-periodic fluctuations in their decreasing order of relevance are $52 \mathrm{yr}$., a group of 3 close to $8 \mathrm{yr}$. (8.8, 8.1 and $8.0 \mathrm{yr}$.), $35.2 \mathrm{yr}$., $18 \mathrm{yr}$. and two near $5.5 \mathrm{yr}$. (5.61 and $5.36 \mathrm{yr}$.). RCs 9 to 12 each individually account for only about 1 percent of the variance with the range of variation of only 5 to 8 in sunspot number (see Fig. 4) and, therefore, are not discussed in detail. The Hale cycle component of $\sim 22 \mathrm{yr}$. manifests only as the 15 th component with an associated period of $20.95 \mathrm{yr}$ and a variance of only $0.5 \%$ (not shown in figure). This is somewhat surprising because Sonett (1982) believes that 10 different periodicities observed in sunspot series can be derived by a simple model involving primarily the $22 \mathrm{yr}$. cycle amplitude modulated by the Gleisberg cycle. The Hale cycle is the manifestation of the changing magnetic field polarity of the sunspot pair. In the $R_{\mathrm{z}}$ time series, only the number of spots and number of groups of spots are taken into consideration. This can explain the minimal influence of the 22-yr. component to the total variance of the time series. It is also likely that the observed Hale cycle is only due to the non-stationarity or the highly intermittant nature of the series and is not a regular feature of the sunspot database. The time variations, shown in Fig. 4 for various periodicities bring out clearly the highly intermittant nature of their amplitude enhancement, lending credence to this inference. This should imply, in turn, that any attempt to predict the time series of individual reconstructed components by any approach can be successful only for a short range of interval in the immediate future. Prediction, too far out into the future will not ever be successful.

Kane and Trivedi (1985) used the MEM directly on the $R_{\mathrm{z}}$ series of 1749 to 1971 identifying the hidden periodicities. They listed some significant oscillations separately for the 1749-1971 and 1845-1971 epochs and found that the two segments have no common periodicity except one with a period of $9.7 \mathrm{yr}$. Their list does not include two of the three fundamental periods identified here except the one close to 11.1 yr. Quasiperiodic fluctuations with 9.7 and 11.0 or 11.1 yr. periodicity were earlier reported by Currie (1973) and Cohen and Lintz (1974). In contrast, Cole (1973) concluded that the $10.45 \mathrm{yr}$ oscillation is a basic mode associated with the excitation of the new solar cycle while a $11.8 \mathrm{yr}$. period could be associated with the free-running length of the solar cycle. He also detected $88 \mathrm{yr}$. and $59 \mathrm{yr}$. periodicities which he attributed to the amplitude modulation of the solar cycle by a period of $11.9 \pm 0.3 \mathrm{yr}$. In our analysis, we do find a clear evidence for these two longer period fluctuations but they appear somewhat shifted in the spectrum (110 yr and $52 \mathrm{yr}$ ). DeMeyer (1981) showed that the sunspot record covering 1749-1977 can be represented adequately by a trigonometric model of 26 different lines. The more important of these with amplitude in excess of 10 units of $R_{\mathrm{z}}$, are 11.11, 90.15, $9.95,11.99,10.58$ and $58.51 \mathrm{yr}$. Interestingly, the significant lines were clustered in four groups $11.11,90.15,8.16$ and $5.79 \mathrm{yr}$. The spectra of the reconstructed components suggest nearly the same order of relative importance.

In conclusion, we suggest that the $R_{\mathrm{z}}$ time series is dominated by the doublet 10.35 and 11.13 yr. a long-period oscillation (110 yr.) and a cluster which may be data dependent with periods close to 8 and $5.5 \mathrm{yr}$. SMC is not a dominant feature of the time series analysed.

\subsection{Prediction of solar activity in cycle 23 (1997-2009)}

As mentioned in the introduction, most of the attempts of predictions of solar activity (summarised in Brown, 1992) aim at providing only the estimated amplitude at the next solar maximum epoch based on either "statistical" or "precursory" approach. Wilson (1988, 1990a, 1990b) utilized the rate of growth of solar activity to predict successfully the solar maximum in the same cycle but the lead time is found to be very short. Wilson (1992) utilized the two major features of the sunspot series (i) an inferred long term increase with time, of the maximum amplitude and (ii) inherently different nature of the even/odd numbered cycles and suggested that cycle 23 would be potentially one of the greatest cycles of modern era, if not the greatest.

If the $R_{\mathrm{z}}$ series is quasi-periodic, then periodic extension can lead to somewhat reliable prediction but it is found that the earlier attempts (DeMeyer, 1981; Kane and Trivedi, 1985 and others) have not been very fruitful. Some investigators have approached the problem through non-linear dynamics or the theory of chaos (Jinno et al., 1995). Application of an attractor analysis has led to the evidence of low-dimensional non linear dynamics (Kurths and Ruzmaikin, 1990). Though chaotic systems are theoretically unpredictable in the long term, the underlying deterministic nature of the systems allows for accurate short term prediction (Mundt et al., 1991). Discussing the predictability of the 
Wolf sunspot number, Sneyers and Cugnon (1986) suggest that autoregressive representation of the series will enable successful short term prediction. It may be recollected that Yule (1927) represented the $R_{\mathrm{z}}$ series as a second order AR process which corresponds to a damped sinusoid and leads to a dominant $10.6 \mathrm{yr}$. periodicity.

We follow the method of adaptive filtering through SSA and low order AR modelling for prediction, as suggested by Keppenne and Ghil (1992). As the prediction beyond a

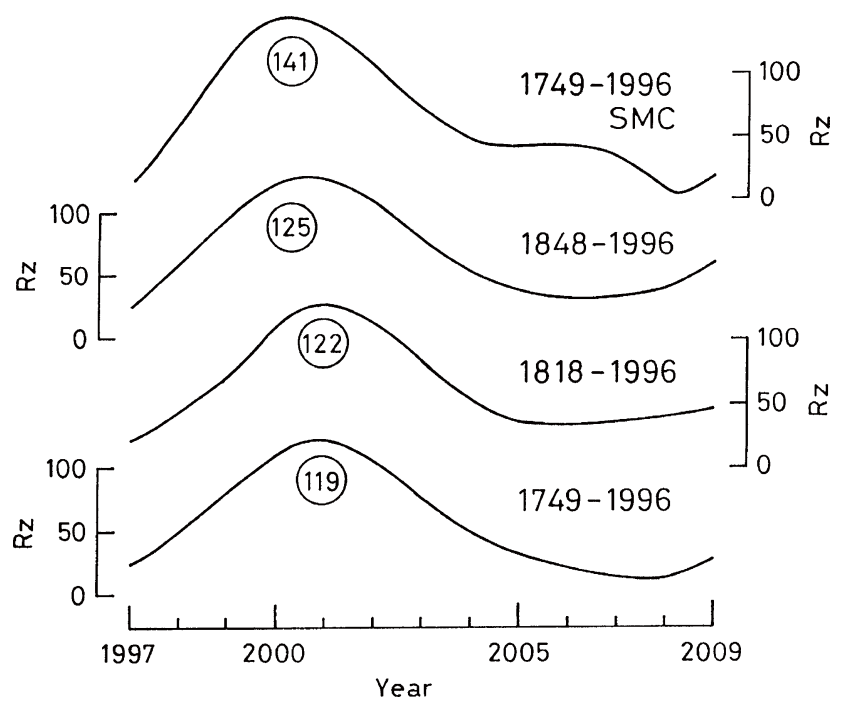

Fig. 7. Predicted variation in annual mean $R_{\mathrm{z}}$ for the next 13 years (19972009) covering solar cycle No. 23. The three sets of data correspond to (i) the entire time series of $R_{\mathrm{z}}: 1749-1996$, (ii) series with "good" and "reliable" data: 1818-1996, and (iii) series with "most reliable" data: 1848-1996. The top curve (SMC 1749-1996) is derived from the series $1749-1996$ by taking $R_{\mathrm{z}}$ in alternate solar cycles with negative magnitudes. Numbers in circles indicate the predicted maximum annual value for $R_{\mathrm{z}}$.

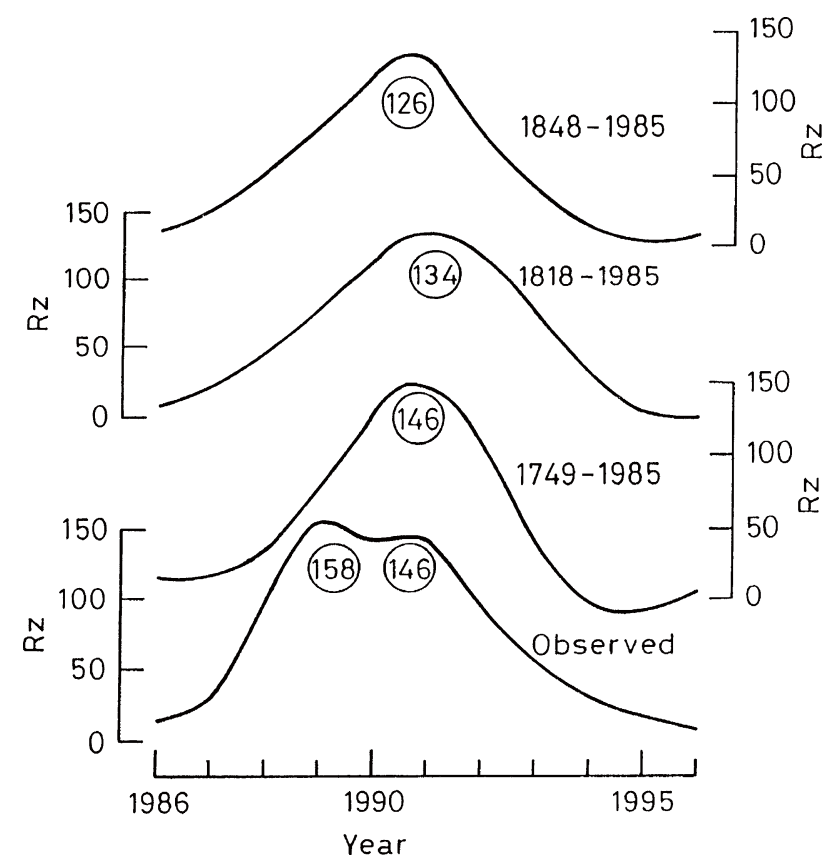

Fig. 8. Observed and predicted variation in the annual mean $R_{\mathrm{z}}$ for solar cycle 22 1986-1996. certain length become unreliable in the AR based approach, we use a weighted average as described in the earlier section.

The 13-point prediction covering the years from 1997 to 2009 is derived from 4 sets, for reasons stated. The time variations of the projected sunspot number covering the next solar cycle (No. 23) is shown in Fig. 7. The three sets of data 1749-1996; 1818-1996 and 1848-1996 all yield practically the same predicted values between 1997 and 2004 but beyond the first 7 years there are differences. The next solar maximum is expected to be in the year 2001 with an anticipated annual mean value of about 122 . The associated uncertainty will be of the order of $5 \%$ due to the contribution of the "noisy" components, not included in the reconstructed series. The rate of decline appears to be slower compared to the ascent, as with almost all the previous solar cycles. The predicted epoch of minimum is, however, not very clearly defined. With a lead time of 5 to 6 years we may be able to give the correct phase of the solar minimum. The similarity of results for 1749-1996 (inclusive of the "questionable" epoch), 1818-1996 and 1848-1996, leads us to confirm that inclusion of the data of first 7 cycles does not significantly alter the conclusions, as suggested earlier by Mundt et al. (1991).

As the model involving the solar magnetic cycle ( $22 \mathrm{yr}$.) amplitude modulated by the Gleisberg cycle ( $\sim 80 \mathrm{yr}$.) can reproduce the $R_{\mathrm{z}}$ spectrum to a large extent (Sonett, 1982), we used the 1749-1996 series modified by negative amplitudes in alternate cycles and computed the forecast values of $R_{\mathrm{z}}$ for the 13 years of cycle 23. This is also shown in Fig. 7. The major differences with respect to the other three approaches are seen to be (i) the epoch of maximum is earlier (close to $2000 \mathrm{AD}$ ), (ii) the amplitude of maximum is larger $\left(R_{\mathrm{Z}}=141\right)$ and (iii) the rate of ascent to maximum and the initial descent from maximum are faster. The prediction beyond $2004 \mathrm{AD}$ appears unreliable once again, as there is an indication of a secondary increase. With a $5 \%$ uncertainty, the lower limit of this forecast and the upper limit of the other three forecasts are nearly the same. Thus an expected value of 130 can be associated with the ensuing solar maximum with its maximum phase in late $2000 \mathrm{AD}$ or early 2001 AD.

This anticipated annual mean sunspot numbers for next seven to 13 years shows that the next solar cycle will not be the greatest or potentially one of the greatest as postulated by Wilson (1992). Cycle 23 will be similar to the cycle 21 with about 4 years in the ascending phase and a reasonably broad maximum over 2 years centred on late 2000 AD.

To establish the relative success of our approach to prediction, the sunspot series for 1986 to 1996 (covering solar cycle 22, for which observations are available) was extrapolated from three different data sets: (i) 1749-1985; (ii) 1818-1985 and (iii) 1848-1985. The predicted solar cycle variations and peak values for the three categories are depicted in Fig. 8 and are compared with the observed annual mean values. We find that the peak value (secondary maximum) and decline from the peak match quite well with observations within error limits. However, the first maximum with a value of 157 is reached more rapidly than the predicted rise and hence defied proper extrapolation. The 
average variation of the sunspot numbers for odd and even cycles over cycles 10 to 21 agrees quite well with the pattern predicted for cycle 22 by the present analysis. Brown also finds that the first maximum in cycle 22 was attained much earlier when compared to the average rise time based on 12 other solar cycles. Wilson (1990a) also found that cycle 22 exhibits anomalous features which could not be predicted by statistical precursor methods. As the extrapolation beyond the range of observations is based on the smooth time variations of individual reconstructed components, any sporadic rise deviating from the anticipated behaviour as noticed during 1988-1989 cannot easily be modelled. Within this constraint, we feel that our forecast for the coming solar cycle will prove to be reliable and hopefully, will be confirmed soon.

\section{References}

Attolini, M. R., S. Cechini, M. Galli, and T. Nanni, On the persistence of 22 year solar cycle, Solar Physics, 125, 389-398, 1990.

Brown, G. M., The peak of solar cycle 22: predictions in retrospect, Ann. Geophys., 10, 453-461, 1992.

Cohen, T. J. and P. R. Lintz, Long term periodicities in the sunspot cycle, Nature, 250, 398-400, 1974.

Cole, T. W., Periodicities in solar activity, Solar Physics, 30, 103-110, 1973.

Currie, R. G., Fine structure in the sunspot spectrum 2 to 70 years, Astrophys. Space Sci., 20, 509-518, 1973.

DeMeyer, F., Mathematical modelling of the sunspot number, Solar Physics, 70, 259-272, 1981.

Dettinger, M. D., M. Ghil, C. M. Strong, W. Weibel, and P. Yiou, Software expedites singular spectrum analysis of noisy time series, EOS Trans AGU, 76(2), 12, 14, 21, 1995.

Dicke, R. H., The phase variation of the solar cycle, Solar Physics, 115, 171-181, 1988.

Dodson, H. W. and E. R. Hedeman, Solar Terrestrial Physics/1970 Part I, edited by C. DeJager, pp. 151-172, D. Reidel, Holland, 1972.

Harwood, J. M. and S. R. C. Malin, Sunspot cycle influence on the geomagnetic field, Geophys. J. R. Astr. Soc., 50, 605-619, 1977.

Hathaway, D. H., R. M. Wilson, and E. J. Reichmann, The shape of the sunspot cycle, Solar Physics, 151, 177-190, 1994.

Jinno, K., S. Xu, R. Berndtsson, A. Kawamura, and M. Matsumoto, Prediction of sunspots using reconstructed chaotic system equations, $J$. Geophys. Res., 100, 14773-14781, 1995.
Kane, R. P. and N. B. Trivedi, Periodicities in sunspot numbers, J. Geomag. Geoelectr., 37, 1071-1085, 1985.

Keppenne, C. L. and M. Ghil, Adaptive filtering and prediction of the southern oscillation index, J. Geophys. Res., 97, 20449-20454, 1992.

Kurths, J. and A. A. Ruzmaikin, On forecasting sunspot numbers, Solar Physics, 126, 407-410, 1990.

MacDonald, G. J., Spectral analysis of time series generated by non linear process, Rev. Geophys. Space Phys., 27, 449-469, 1989.

Marple, S. L., Jr., Digital Spectral Analysis with Applications, pp. 232236, Prentice Hall, New Jersey, 1987.

McKinnon, J. A., Sunspot numbers 1610-1985 UAG Rep. 95, pp. 112, NOAA Boulder, Colorado, U.S.A., 1987.

Mundt, M. D., W. B. Maguire, II, and R. R. P. Chase, Chaos in the sunspot cycle: Analysis and prediction, J. Geophys. Res., 96, 1705-1716, 1991.

Pasricha, P. K., S. Aggarwal, and B. M. Reddy, Model evaluation of the year-to-year variability in a 11-year sunspot cycle, Ann. Geophys., 9, 696-702, 1991.

Penland, C., M. Ghil, and K. M. Weickmann, Adaptive filtering and maximum entropy spectra with application to changes in atmospheric angular momentum, J. Geophys. Res., 96, 22659-22671, 1991.

Rabin, D., R. M. Wilson, and R. L. Moore, Bimodality of the solar cycle, Geophys. Res. Lett., 13, 352-354, 1986.

Sneyers, R. and P. Cugnon, On the predictability of the Wolfe sunspot number, Ann. Geophys., 4, 81-86, 1986.

Sonett, C. P., Sunspot time series spectrum from square land modulation of the Hale cycle, Geophys. Res. Lett., 9, 1313-1316, 1982.

Ulrych, T. J. and T. N. Bishop, Maximum entropy spectral analysis and autoregressive decomposition, Rev. Geophys. Space Phys., 13, 183-200, 1975.

Vautard, R., P. Yiou, and M. Ghil, Singular spectrum analysis: a toolkit for short, noisy chaotic signal, Physica, D58, 95-126, 1992.

Wilson, R. M., Predicting the maximum amplitude for the sunspot cycle from the rate of rise in sunspot number, Solar Physics, 117, 179-186, 1988.

Wilson, R. M., Predicting the maximum sunspot number: a comparative study between single variate and bivariate precursor techniques, Solar Physics, 125, 143-145, 1990a.

Wilson, R. M., On the average rate of growth in sunspot number and the size of the solar cycle, Solar Physics, 125, 133-141, 1990b.

Wilson, R. M., An early estimate for the size of cycle 23, Solar Physics, 140, 181-193, 1992.

Yule, G. U., On a method for investigating periodicites in disturbed series with special reference to Wolfe's sunspot number, Phil. Trans. R. Soc. London A, 226, 267-298, 1927.

G. K. Rangarajan (e-mail: ranga@iig.iigm.res.in) 